$\mathbb{P}$ periodica polytechnica

Civil Engineering

57/2 (2013) 201 210

doi: 10.3311/PPci.7175

http://periodicapolytechnica.org/ci

Creative Commons Attribution (1)

RESEARCH ARTICLE

\section{Passenger number dependent traffic control in signalized intersections}

\author{
János Polgár / Tamás Tettamanti / István Varga
}

Received 2012-09-29, revised 2013-08-23, accepted 2013-09-16

\begin{abstract}
The paper investigates a novel approach to the traffic control problem of signalized junction with bus priority. In road traffic engineering practice, adaptive control algorithms are generally used for maximizing the traffic flow of intersections. The adaptive strategies aim to calculate optimal signal splits for the traffic lights. The calculation is usually based on the number of vehicles measured by appropriate detection methods and may also depend on public transport priority actions (e.g. "green call" by buses). The main drawback of these methods is that the number of vehicle passengers is not taken into consideration. Unlike the common approaches the presented control strategy considers weighted passenger number exclusively (by using the assumption that the number of passengers can be measured in all vehicles). Hence, the control aim is to maximize the number of weighted passenger number capable to cross the intersection. The proposed control method is based on two factors: the number of passengers in road vehicles and their temporal distance measured from the stop line. The strategy does not apply priority measures for public transport directly. At the same time, the vehicles carrying numerous passengers (i.e. buses) acquire preference.
\end{abstract}

\section{Keywords}

road traffic control $\cdot$ bus priority $\cdot$ weighted passenger number

\section{János Polgár}

Department of Aerospace Engineering and Mechanics, University of Minnesota, MN 55455, Minneapolis, USA

e-mail: polgx001@umn.edu

\section{Tamás Tettamanti}

Department of Control for Transportation and Vehicle Systems, Budapest University of Technology and Economics, Stoczek u. 2., H-1111 Budapest, Hungary e-mail: tettamanti@mail.bme.hu

\section{István Varga}

Department of Control for Transportation and Vehicle Systems, Budapest University of Technology and Economics, Stoczek u. 2., H-1111 Budapest, Hungary

Systems and Control Laboratory, Computer and Automation Research Institute, Hungarian Academy of Sciences, Kende u. 13-17., H-1111, Budapest, Hungary

e-mail: ivarga@mail.bme.hu

\section{Introduction}

One of the most important elements of sustainable transportation is the development of public transport, i.e. searching methods which ensure advantages for public vehicle passengers. Traffic congestions in big cities can be observed day-by-day as a permanent problem. Without any detached track or priority bus lanes [1,7] the public transport vehicles are forced to run on congested roads in peak hours. Therefore, public transport may become unpredictable for the passengers and the transportation company as well. To avoid this problem bus priority can be used in signalized intersections [16].

The proposed traffic control method applies a novel approach to give priority for public transport vehicles. The strategy considers the passengers in the vehicles; moreover the position of these passenger groups is taken into account as well. Thus, the bus priority and the demand of private cars are handled together in an indirect way. The control aim is to maximize the weighted passenger number which crosses the intersection during the cycle time. This approach requires special infrastructural elements concerning the detection of the vehicle passengers. Nevertheless, such detection systems exist in practice and imply a promising technology for the future intelligent transport systems.

The first sections of the paper introduce the preliminaries and methodology of passenger number dependent traffic control. In the ensuing parts, control algorithm and feasibility questions are investigated. Finally, simulation and results are presented and evaluated.

\section{Preliminaries}

In the practice of road traffic management different control actions exist to give priority for buses. Formerly, the passive actions were generally used. Nowadays, the active priority measures are rather applied [5, 6, 11]. There are four commonly used active actions that a traffic controller can perform in response to the detection of a public transport vehicle: extension of the green interval in the current stage, ending another stage earlier to give an early green to the vehicle, inserting an extra stage to allow the vehicle to pass before returning to the regular 
signal plan and stage rotation to attain the priority stage earlier. In most cases, the action of green extension is used by reason of its simplicity. We showed in [13] that these measures without restrictions can be efficiently applied if only a few buses may arrive to the intersection at the same time. Moreover, if the overall traffic increases the effectiveness of the classical bus priority may reduce significantly. Hence, a novel approach is introduced called as passenger number dependent traffic control.

There are plenty of developed strategies in the literature, which are able to give priority for public transport vehicles. Without the pursuit of completeness we refer numerous articles. The widely used SCOOT system has a built-in transit priority function [3]. The algorithm of the TRANSYT-7F model minimizes a performance index to achieve optimal cycle lengths as well as green time splits [14]. The performance index contains weighting factors regarding the road links. Thus, the links used by buses can be properly weighted to assure transit priority. Reference [2] presents a priority system based on the estimated arrival time to the stop line of the buses. Paper [9] investigates a strategy using real time GPS data to determine the position of the public transport vehicles. The public transport system can be considered as a state space model known from the modeling theory. Article [4] proposes hybrid predictive control formulation to optimize the real-time operations of buses. Standard bus priority as well as the avoiding of a second stop of the bus at the end of the queue are demonstrated by numerical examples in [8]. Similarly to our strategy the RHODES/BUSBAND approach applies weights to give priority for buses. However, unlike the proposed concept in our paper, it calculates the weights depending on the delay of the vehicle and the passenger number [10].

\section{Control parameter of the strategy}

The basic goal of our strategy is to split the available green time among the signal stages of the intersection in order to maximize the number of passengers crossing the stop line. The method is based on the time weighted passenger numbers (abbreviated as TWPN in the sequel), called as control parameters.

\subsection{Generating the control parameter}

The vehicles waiting in the branches of the junction (intending to pass through the junction) form passenger groups. The crossing time of these passenger groups fundamentally depends on the distance measured from the stop line. More exactly, it does not depend on the spatial distance but on the temporal distance, i.e. the time needed to cross the stop line.

The passing time of the passenger groups can be defined based on the approximated saturation flow rate, e.g. about 0.5 vehicle/s as commonly used in practice. This means that in every 2 seconds a vehicle crosses the stop line. By considering this general approach, the passenger groups may pass the branch of a junction in every 2 seconds if their vehicles are arriving continuously. Naturally, in case of buses or successive vehicles with larger following distance, smaller saturation flow rate must be applied.

By knowing the temporal distance (calculated based on the saturation flow described above) and the passenger number of each vehicle in all branches, the control parameters can be calculated as the product of them, called as time weighted passenger numbers. This parameter serves as the basis of the proposed traffic control approach. Practically, each vehicle is substituted by a weighted passenger number in the following considerations.

Similarly to the vehicles, these numbers can be virtually positioned behind the stop line. The distance measured from the stop line can be spatial and temporal as well. However, the traffic signal plans cannot depend on the spatial distance itself. Therefore, a conversion to time basis is required. By the aid of the saturation flow, it is a straightforward calculation process.

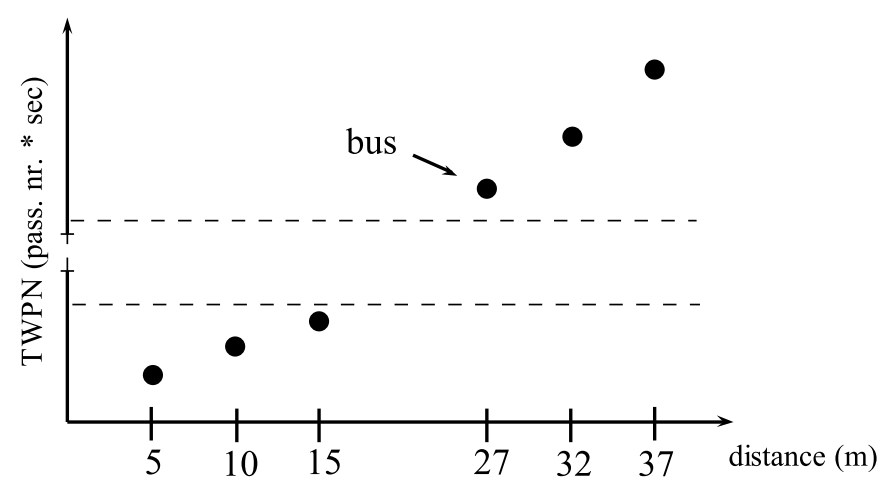

a)

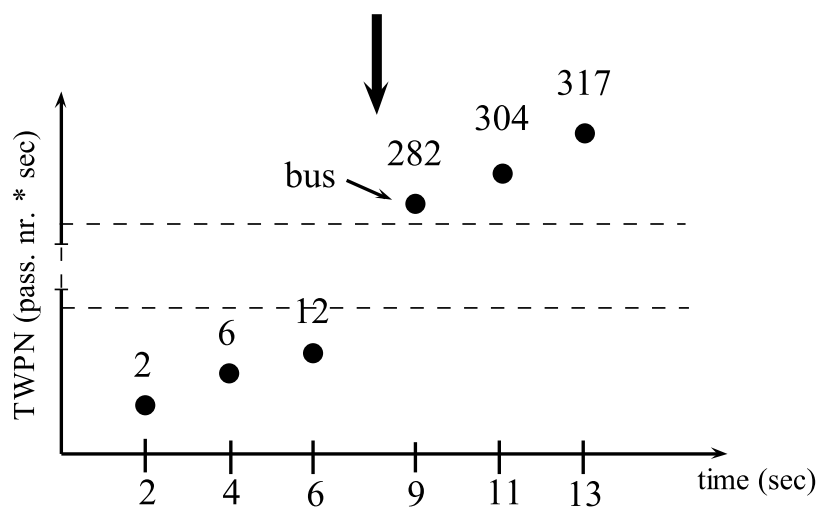

b)

Fig. 1. Derivation of control parameter

The TWPNs are cumulated in each branch in such a way that each TWPN is substituted by the sum of the previous TWPNs plus the TWPN at the given position. The diagrams in Fig. 1//a and 1, $\mathrm{b}$ represent the spatial and temporal positions of cumulated TWPNs in a branch, respectively. The diagrams represent the presence of a bus in the queue as well. This is the reason for the bigger step from the third to fourth data point compared to the others.

By the creation of the above diagrams several theoretical assumptions were introduced. For the sake of simplicity the passengers are situated at the back of the vehicles. Moreover, only 
integer seconds are considered on the time axis, which requires the rounding of the TWPN in certain cases. The spatial representation was used only for demonstration purposes. Only the diagram on temporal basis will be applied in the sequel.

Further calculation is required to apply the diagram in Fig. 1] b in the control process as the traffic controller needs a control parameter at each second. Some TWPNs, however, are missing at certain seconds between the first and last points of the diagram (see Fig. 1] b), since the vehicles cannot pass the intersection at every second. In this case, the missing values can be replaced by the point of the previous time value. This practical operation does not cause any problem since no passenger group can be situated between two distinct points. Thus, the same quantity of passengers can cross the stop line at the newly inserted points as at the previous one. In the example shown by Fig. 11 b the passenger groups are sitting in common private cars if the previous control parameter precedes by two seconds. Between 6 and 9 seconds, however, a bus can be observed as it needs more passing time by reason of the vehicle length. On the other hand, such phenomena could also be caused by a car if it maintains "too" safe following distance.

An example is represented by Fig. 2 where the measured set of points were augmented to obtain an array containing a point at each time step. For instance, the second car in the queue needs 4 seconds to cross the stop line. The next one behind it can appear at the stop line after 6 seconds. The control algorithm, however, requires data at 5 seconds as well, thus the same TWPN value is assigned to this time step, as the previous one has.

It has to be noted that the diagram of the control parameter is not an exact function but a set of discrete points. One completed point set can be assigned to each branch (i.e. to one signal group) of the intersection. If a stage of the signal plan contains a green time for more than one signal group, the TWPNs can be summed at each second. Hence, the number of point sets is reduced to the number of the stages by this step.

Let us consider the time on the axis $\mathrm{x}$ of the completed diagram as green time. Thus, a point represents the amount of TWPNs, which may pass the intersection in this stage, if the green time indicated on the axis $\mathrm{x}$ is applied. The TWPN based control algorithm applies the introduced completed diagrams (see Section 47. Nevertheless, we present a small-scale example to delight the control parameter in the following subsection.

\subsection{Illustration example}

The completed diagrams of traffic parameters can be represented together. Thus, a control diagram can be created which is the base of the proposed control strategy. Consider a simple two-stage intersection represented by Fig. 3 In this case, the traffic control consists of stage 1 and 2 having green time 1 and 2, respectively. If we are able to detect the passenger groups (with positions) waiting in the two branches of the junction the diagrams of the weighted passenger number can be calculated for each stage. Moreover, after completing the diagram (as described above), a three dimensional representation can be achieved as shown in Fig. 4

The horizontal axes represent the green times of the stages 1 and 2. Based on the control diagram one is able to determine the optimal green times for stage 1 and 2, i.e. the maximal throughput of the junction. This optimal choice denotes one point among the control parameters of the diagram. Nevertheless, the control action must satisfy green time constraints.

Obviously, the increase of the number of stages augments the dimension of the control diagram. In case of more than two stages, the control diagram is not representable visually but still can be used for control calculation. The details of the control algorithm are presented in the following part.

\section{TWPN dependent traffic control}

General properties of the control algorithm are summarized as follows. It applies fix cycle time. Hence, constraints must be considered by the calculation of the length of green times. Minimal and maximal green time intervals are always defined for the stages. Furthermore, a maximal value concerning the sum of the green times has to be taken into account as well.

Note that the control aim is to find the optimal green time combination in which the greatest number of TWPNs can pass the intersection. One could imagine this process using Fig. 5 Move the horizontal lines along the time axis such a way that the indicated TWPNs together result the greatest sum, while the sum of indicated green times equals to the overall green time determined by the intersection geometry and cycle time. The implemented algorithm, however, does not use this method. It rather applies 'brute force' as we can see in the sequel.

The real green time calculation is summarized by the flowchart of Fig. 6. Initially, the algorithm selects all possible green time combinations satisfying the constraints. A combination contains the same number of green time lengths as the number of stages. Since the constraints are predefined, the combinations also can be calculated in advance, independently from the cyclic change of the green time lengths.

In the first step, the completed TWPN diagrams of all stages of the junction have to be calculated at the end of each cycle. Then, for each possible green combination the algorithm sums up the TWPN values the following way. The TWPN value is taken from the $i$ th completed diagram measured at the ith green time of the combination.

In the next step it goes through all the summed TWPNs, and chooses the maximum. The green time combination belonging to this value will be applied in the next cycle.

It may happen that more than one green combination would result the same TWPN representing the overall greatest value for the next cycle. In this special case, the algorithm computes the variance of green times for each competitive combination. The one is chosen which has the smallest variance. Thus, as an output of the control algorithm, the green time intervals of the assigned combination are applied for the next control cycle. 


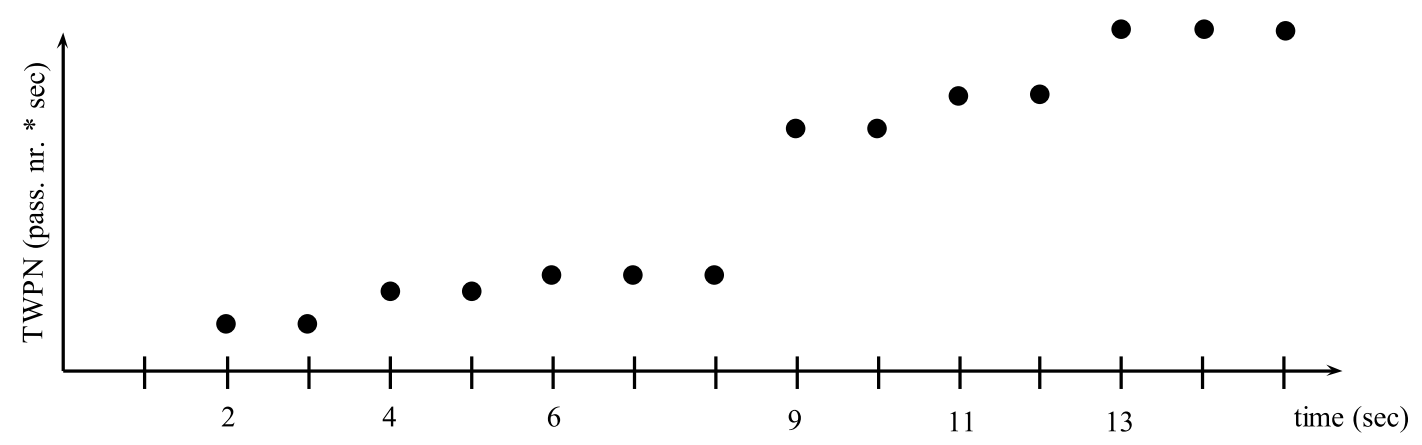

Fig. 2. TWPN in one branch of an intersection

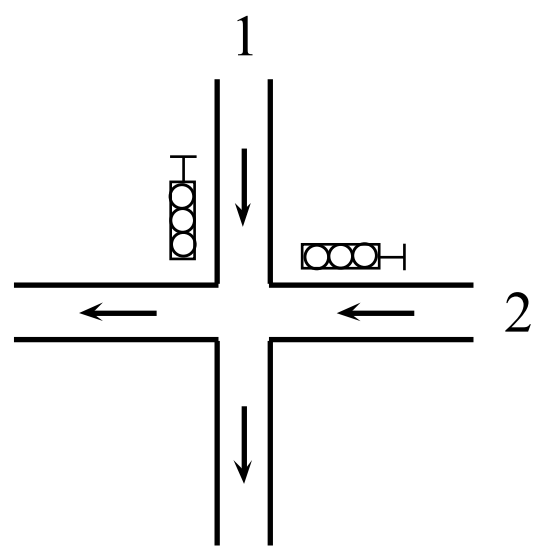

Fig. 3. Two-stage intersection

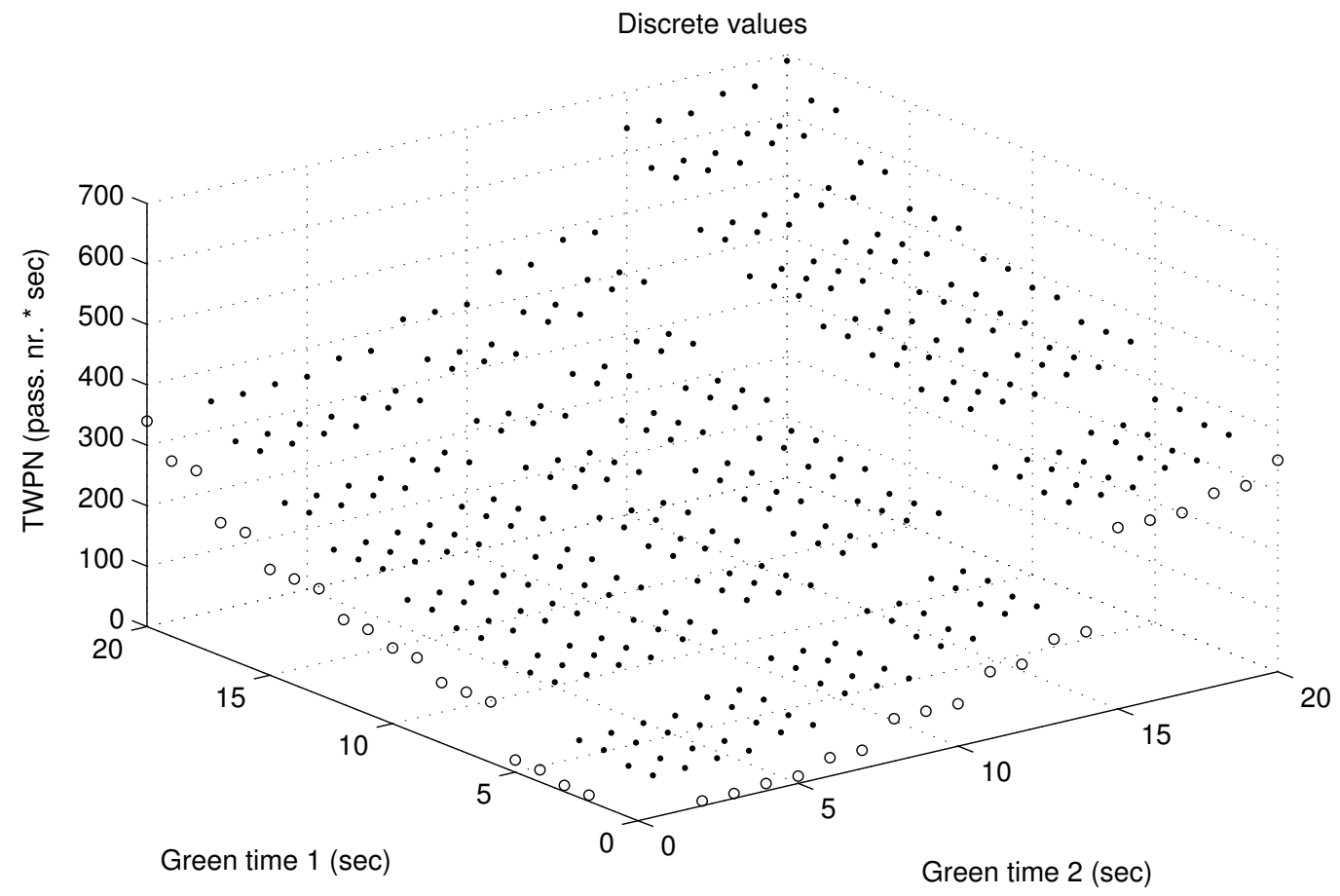

Fig. 4. Control diagram of a two-stage intersection 


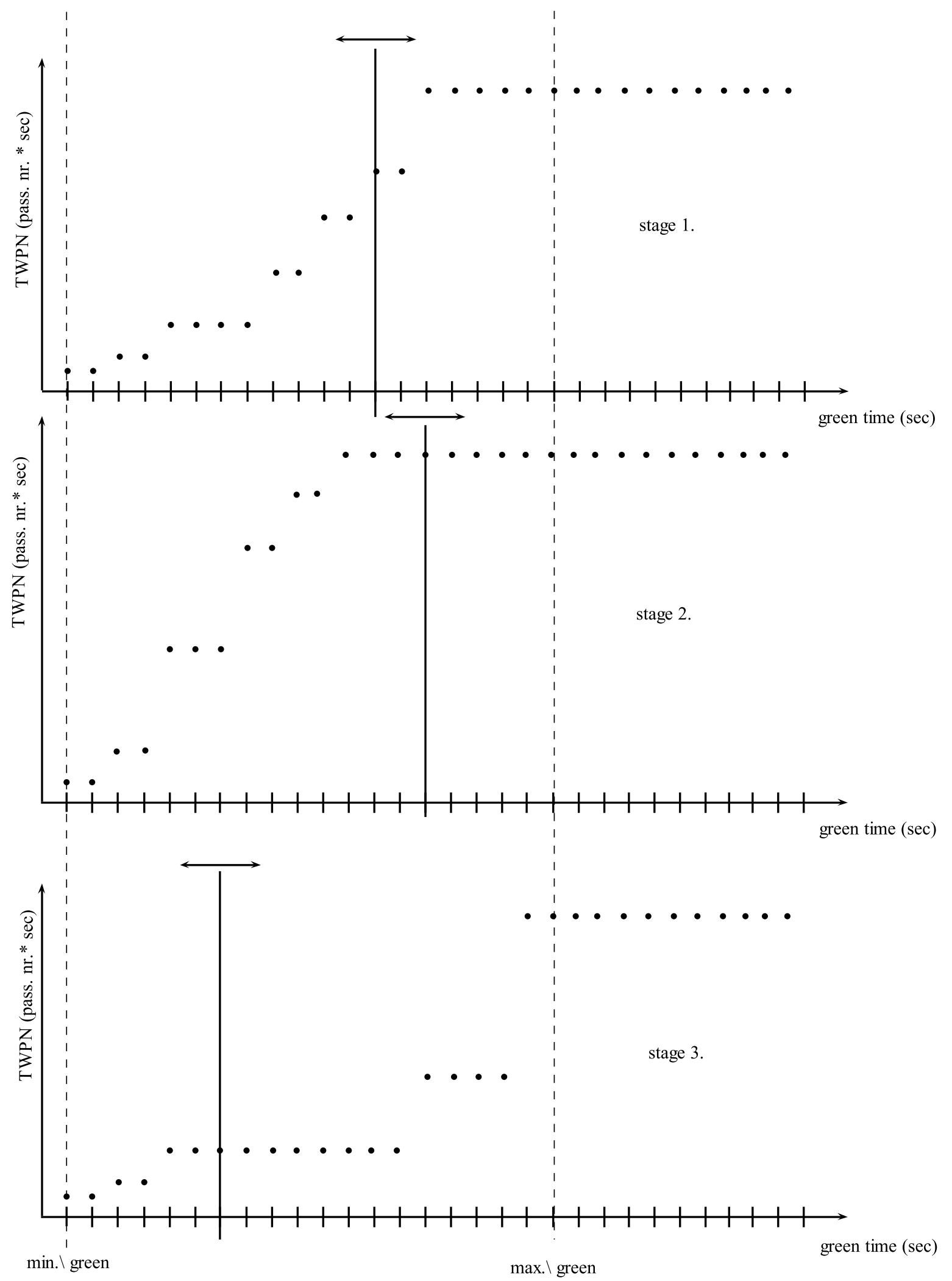

Fig. 5. Illustration of green time calculation in control law 


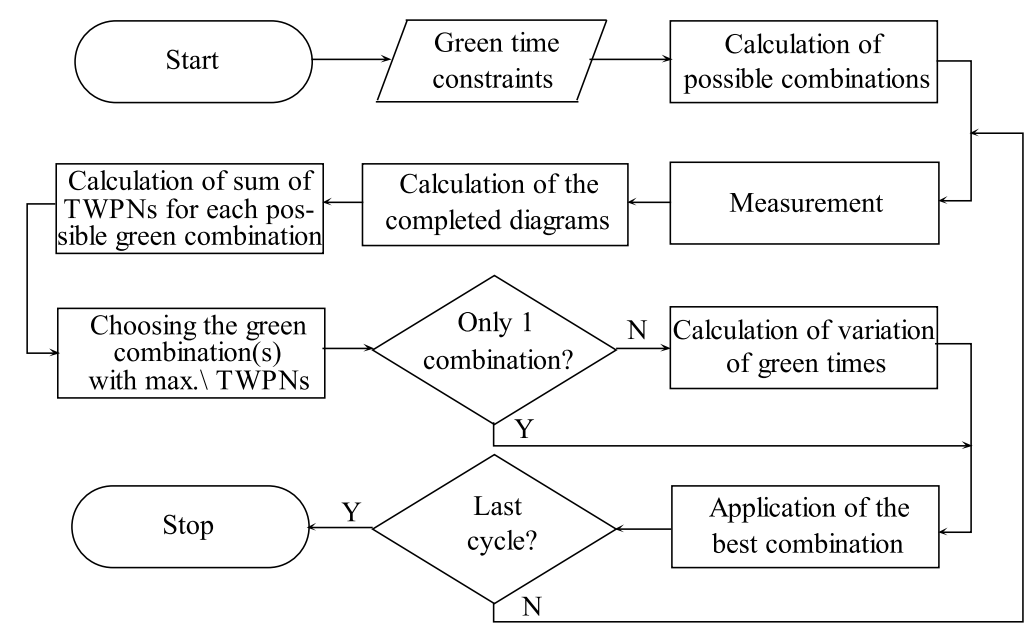

Fig. 6. Flowchart of the control algorithm

\section{Feasibility of the control algorithm}

The feasibility of passenger number dependent control is primarily based on the available measurements. Generally, the adaptive traffic control focuses on the measurement of vehicles. Measurement of the vehicle passengers, however, requires special infrastructure with suitable algorithm. Several solutions exist to count the passengers on buses. One of the most common methods is the detection by the air suspension system. By using a special instrument built in the air springs, the current weight of the bus can be measured. Hence, the number of passengers can be estimated as well. Further possible solutions are the use of photocells at the bus doors or the measurement of pressure by sensors in the stairs. In case of electronic fare payment, RFID technology can also be applied.

The passenger number in private vehicles cannot be detected by these methods. Nevertheless, infrared technology can be applied for passenger counting efficiently. One of the first investigations of this field is provided by [12]. Furthermore, companies exist providing such special infrared camera. An example is the product of [15], which is capable to detect the sitting passengers in private cars independently from weather or light conditions. The instrument has $90 \%$ reliability if the vehicles have a velocity not more than $128 \mathrm{~km} / \mathrm{h}$, and are within a $50 \mathrm{~m}$ distance from the instrument. The camera applies two infrared beams with different wavelengths and creates a digital picture of the vehicle interior. Finally, a special algorithm counts the passengers based on the digital pictures.

Obviously, the infrared technology means an expensive infrastructure, especially in case of larger intersection with several lanes. At the same time, the passenger number on buses can be counted with smaller investment. Moreover, passenger detectors are often used in public buses by the transportation company for statistical reason. Accordingly, the detection of car passengers can be replaced by cheaper solution (e.g. loop detectors for vehicle count) and by using an average passenger number per car, which is about 1.2-1.5. The simulation results (presented later in Section 6) show that the slight inaccuracy of this approach does not influence significantly the overall effectiveness of the control strategy.

\section{Simulation}

The proposed traffic control strategy has been tested in VISSIM simulation environment [18]. The simulator is able to model single intersections as well as network in a microscopic level. VISSIM offers a user friendly graphical interface (GUI) through of which one can design the geometry of any type of road networks and set up simulations in a simple way. However, for several problems the GUI might be not satisfying. This is the case, for example, when the user aims to access and manipulate VISSIM objects during the simulation. For this end, an additional interface [17] is offered based on the COM (a technology to enable the communication between software). Accordingly, COM interface programming (in $\mathrm{C}++$ ) was applied to realize the control algorithm and the passenger detection in VISSIM environment.

\subsection{Test intersection}

The simulations were realized by using a fictional test intersection represented in Fig. 7. The signal plan of the junction has 3 stages with dedicated directions. The branches belonging to the stages are numbered in the figure. All lanes are controlled by traffic signal heads. Movements only from one direction are assigned to stages 1 and 3. Stage 2, however, contains two branches corresponding to the directions of north-east and south-west. Thus, the time weighted passenger numbers belonging junction branches in stage 2 will be summed in one control diagram during the control process.

Two bus lines crossed the test intersection (represented by dotted lines in the figure) in the simulations. Transit vehicles were arriving from the directions of stage 1 and 2, i.e. the lines operate only in one direction: westbound and northeastbound, respectively. Bus stop was not modeled near by the junction. Nevertheless, passengers were always simulated in the vehicles. Buses coming from direction 1 had a service frequency of 6 minutes. The vehicles of the other transit line (arriving from direc- 
tion 2) run every 10 minutes. Thus, the test intersection realized a relatively busy traffic of buses.

We supposed that the measurement system is able to identify vehicles $100 \mathrm{~m}$ beyond the stop line in each branch of the intersection.

\subsection{Simulation scenarios}

The simulation scenarios can be sorted into three groups. The first group of simulations was run with a fix time signal plan. The results of the passenger number dependent control are compared to those of the first group of scenarios. The second group of simulations was run with the proposed control strategy using identical green time constraints for all stages: green time intervals could change from 4 to $20 \mathrm{~s}$. The third group of simulation scenarios is similar to the previous one. It only differs in the constraints: green time was allowed from 4 to $30 \mathrm{~s}$. The signal control used fixed stage order and fixed cycle time (60 s) in all simulation scenarios.

As VISSIM simulator operates in a deterministic way two simulation runs using identical parameter settings provide the same results. To avoid this effect a random seed parameter can be changed which influences the distribution of the arrival of the traffic inflow. By changing the random seed, different traffic situations arise in the simulation. Each scenario has run with five different random seeds. Thus, the results presented in the tables of the following parts are always averaged from five runs per scenario.

The traffic flow demand of the three stages was changed in each scenario. Nevertheless, the ratio among them was kept identical. For simplicity, the passenger number of buses was the same ( 80 person) in all transit vehicles crossing the intersection. The occupancy of the private cars was set to 1.3 passengers per car in average.

The simulation time was one hour long in all scenarios.

\section{Simulation results}

The passenger number dependent control was evaluated compared to the fix time signal strategy based on the average traffic demand, i.e. the number of vehicles per hour. According to the two different strategies, the fix time signal plans were generated with $20 \mathrm{~s}$ and $30 \mathrm{~s}$ maximal green time constraints, and naturally for all combinations of traffic input flows used in the simulations.

Table 1 and 2 show the simulation results of the proposed strategy using maximum $20 \mathrm{~s}$ and $30 \mathrm{~s}$ long green times, respectively. The relative variation of traffic parameters compared to the fix time strategy resulted by the simulations with passenger number dependent control are shown. The first column of the tables contains the predefined input traffic flows of stages 1,2 and 3 (the flows of the directions of stage 2 are summed), respectively. Nevertheless, it has to be noted that the input flow (set by the user) cannot be generated in the simulator by all means. It may happen that the whole quantity of the predefined vehicles cannot enter the traffic network if there is no space on the current link by reason of heavy traffic.

The relative variation of three different traffic parameters was analyzed based on the simulation results. The first parameter to evaluate is the passenger number which gives the number of travelers capable to pass the intersection. The variation of the passenger numbers resulted by the new strategy did not changed significantly. It is due to the fact that the number of public buses was the same in all scenarios with the same schedule. Therefore, significant enhancement was not possible in the simulations. The second observed parameter is the passenger-hour calculated for all vehicles of the simulation. Passenger-hour is calculated as the product of the passenger number and the travel time spent in the network by a given vehicle. The third traffic parameter evaluated in Table 1 and 2 the number of stops (NS) which sums the vehicle stops occurred in the network. Similar values were obtained with both green time lengths regarding the second and third evaluation parameters. Hence, we discuss the trends and conclusions of both tables together.

Generally we can assert that the TWPN based control strategy has benefits for buses, nevertheless the traffic conditions of car drivers worsened significantly. The results have similar characteristics to our initial expectations. The phenomena, however, is compensated by the good results of buses. The passenger-hour parameter of buses decreased at each simulation case at least with $7 \%$. Buses produced a striking diminish in point of the parameter number of stops. More than $50 \%$ decrease was also measured. In case of the cars we find positive value in almost all entry of the tables. A slight trend of increase can be observed in the car concerning columns of Table 1 .

Unfortunately the simulation results show unpredictable fluctuation, which caused by the simulation software. In some traffic situation (e.g. overtaking) the software cannot follow the way of thinking of the real drivers. Therefore, the simulation results in the slowing down of the traffic with many stopping and restarting. These cases can significantly worsen the statistical data, especially in case of peak traffic, as represented by the four last lines of Table 1 and 2 A trade-off arises in these cases. Although, the results regarding the cars fell off significantly, the headway of the buses was improved considerably by the application of the TWPN based control strategy.

Beside the relative values of the Table 1 and 2 the results of total time spent in the network (abbreviated as TTS) resulted by the simulation runs with $20 \mathrm{~s}$ maximum greens are shown in Fig. 8 and 9, for buses and cars respectively. TTS expresses the sum of the waiting and travel times of all the vehicles from one class. The trend of the achieved TTS parameters is similar to that of the passenger-hour, i.e. this reflects the same results as presented in the tables.

The fixed time strategy vs. TWPN based method comparison has already been performed in the previous paragraphs, thus we focus on the absolute value. In case of buses the TTS did not increased significantly as the traffic flow rose. Of course, there 


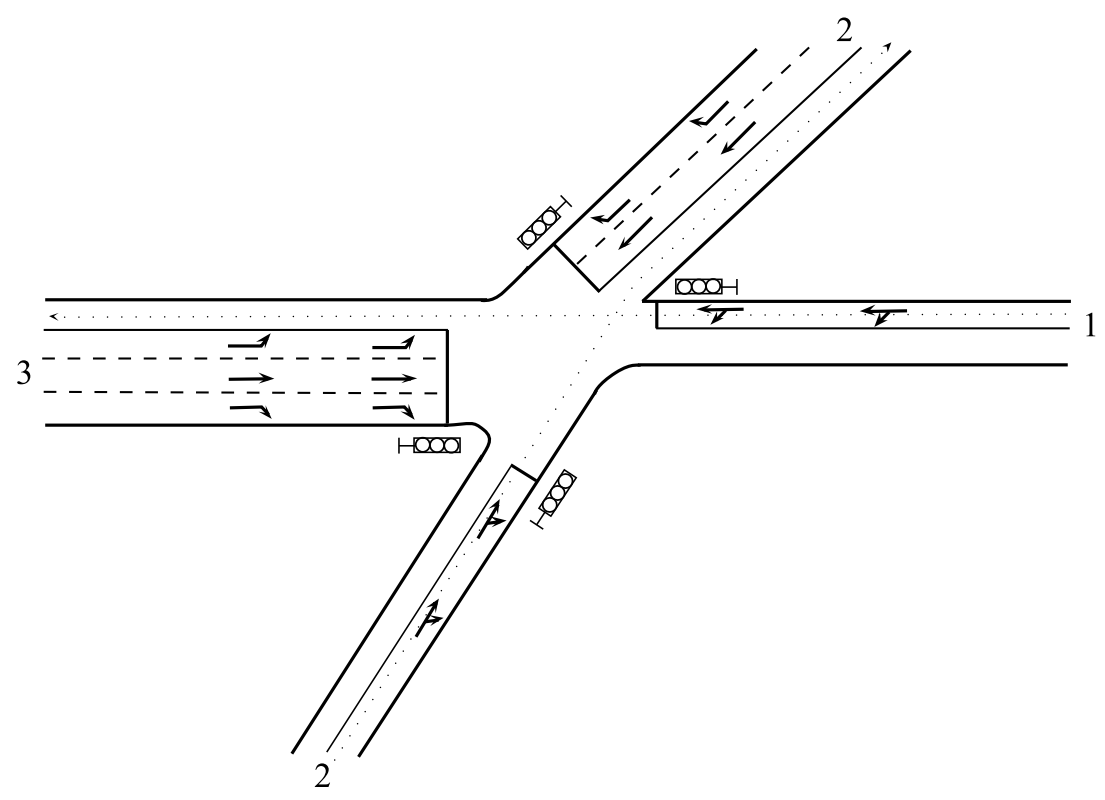

Fig. 7. Three-stage test intersection

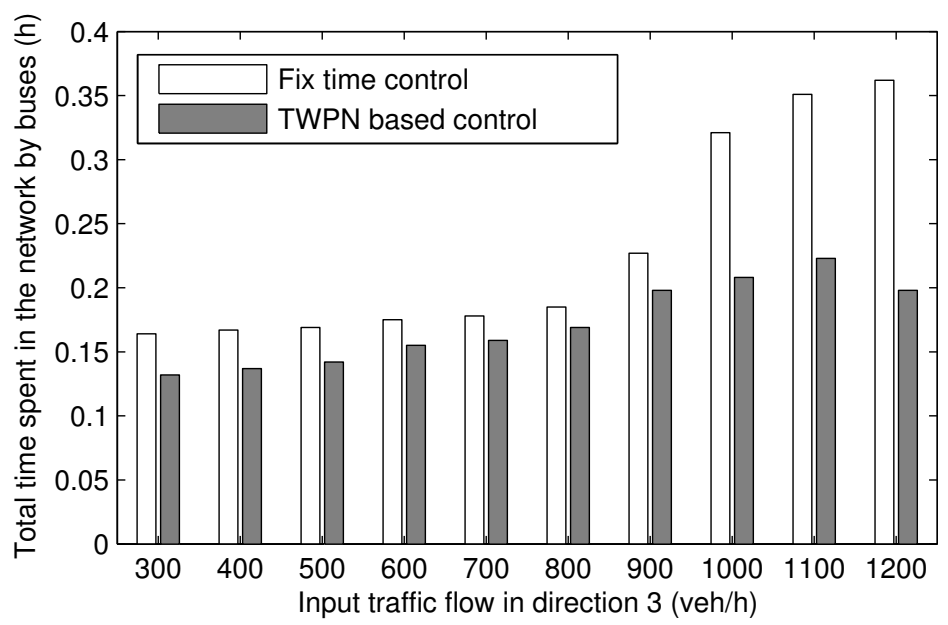

Fig. 8. TTS parameter of buses resulted by the simulation runs with $20 \mathrm{~s}$ maximum

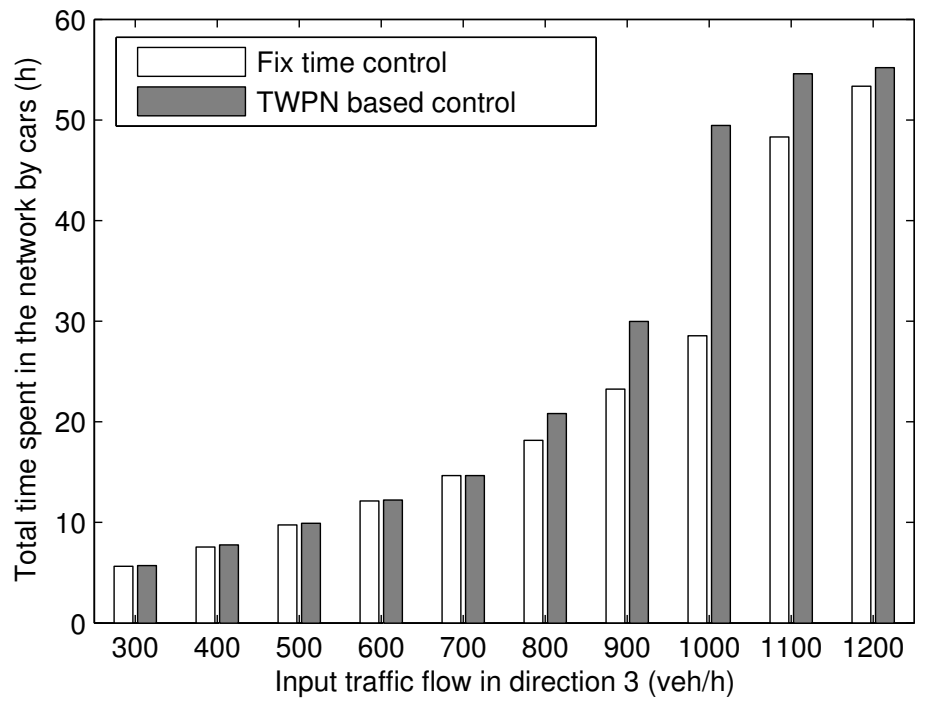

Fig. 9. TTS parameter of cars resulted by the simulation runs with $20 \mathrm{~s}$ maximum green times 
Tab. 1. Parameter variations with $20 \mathrm{~s}$ maximum allowed green times

\begin{tabular}{lccccc}
\hline & \multicolumn{5}{c}{ Max 20 s green times } \\
\cline { 2 - 6 } & $\begin{array}{c}\text { Variation of } \\
\text { passenger } \\
\text { number (\%) }\end{array}$ & Variation of passenger-hour (\%) & Variation of number of stops (\%) \\
\hline $\begin{array}{c}\text { Input traffic flow } \\
\text { (veh/h) }\end{array}$ & bus+car & bus & car & bus & car \\
\hline $150 / 450 / 300$ & -0.01 & -19.32 & 0.76 & -36.25 & 0.12 \\
\hline $200 / 600 / 400$ & -0.02 & -17.51 & 2.08 & -30.00 & 4.72 \\
\hline $250 / 750 / 500$ & -0.05 & -15.76 & 1.31 & -26.25 & 1.33 \\
\hline $300 / 900 / 600$ & -0.09 & -12.84 & 1.60 & -17.50 & 0.13 \\
\hline $350 / 1050 / 700$ & -0.05 & -10.17 & -19.77 & -15.00 & -0.48 \\
\hline $400 / 1200 / 800$ & -0.04 & -7.83 & -6.45 & -7.32 & 19.13 \\
\hline $450 / 1350 / 900$ & -1.34 & -8.68 & 6.33 & -6.45 & 44.18 \\
\hline $500 / 1500 / 100$ & -2.41 & -32.60 & 36.39 & -26.61 & 112.15 \\
\hline $550 / 1650 / 1100$ & -1.47 & -30.81 & -1.08 & -35.56 & 16.5 \\
\hline $600 / 1800 / 1200$ & 0.27 & -46.55 & -13.30 & -50.74 & 9.81 \\
\hline
\end{tabular}

Tab. 2. Parameter variations with $20 \mathrm{~s}$ maximum allowed green times

\begin{tabular}{lccccc}
\hline & \multicolumn{5}{c}{ Max 30 s green times } \\
\cline { 2 - 6 } & $\begin{array}{c}\text { Variation of } \\
\text { passenger } \\
\text { number (\%) }\end{array}$ & Variation of passenger-hour (\%) & Variation of number of stops (\%) \\
\hline $\begin{array}{c}\text { Input traffic flow } \\
\text { (veh/h) }\end{array}$ & bus+car & bus & car & bus & car \\
\hline $150 / 450 / 300$ & -0.01 & -19.32 & 1.07 & -36.25 & -0.46 \\
\hline $200 / 600 / 400$ & 0.02 & -17.84 & 2.61 & -28.75 & 3.84 \\
\hline $250 / 750 / 500$ & 0.04 & -15.84 & 1.62 & -26.25 & 0.75 \\
\hline $300 / 900 / 600$ & -0.09 & -11.54 & 0.69 & -18.75 & 0.59 \\
\hline $350 / 1050 / 700$ & 0.98 & -10.57 & -0.02 & -15.00 & -21.70 \\
\hline $400 / 1200 / 800$ & 1.03 & -8.43 & 14.88 & -7.32 & -4.66 \\
\hline $450 / 1350 / 900$ & -0.08 & -12.86 & 29.04 & -2.25 & 18.47 \\
\hline $500 / 1500 / 100$ & -1.91 & -35.12 & 67.30 & -26.61 & 63.51 \\
\hline $550 / 1650 / 1100$ & -0.66 & -36.60 & 12.74 & -30.95 & 5.041 \\
\hline $600 / 1800 / 1200$ & 0.35 & -45.25 & 3.48 & -52.82 & -9.17 \\
\hline
\end{tabular}


is a slight rising trend in this parameter, but our control algorithm successfully shortened the time what buses spent on the network. Nevertheless car drivers pay the price of this result. As one can see in Fig. 9 the TTS of this vehicle class increased as more and more vehicles were forced onto the network.

As a conclusion of the simulation, it can be stated that the passenger number dependent strategy improves the system performance compared to the fix time (and vehicle demand based) strategy. Although the parameters of cars decreased in peak traffic situations, the headway of the buses was improved in all simulation cases. Therefore, the aim of the control is achieved.

Naturally, in case of real-word realization several technical aspects must be taken into consideration depending on the intersection geometry and the number of stages, e.g. cycle time, green time constraints.

\section{Conclusions and future development possibilities}

The paper presented a novel strategy for intersection traffic control providing optimal green time splits for signal stages. The control is based on the time weighted number of vehicle passengers, i.e. the temporal distance from the stop line. The strategy was tested by VISSIM simulation software with two different green time constraint settings. The simulation results represent that the proposed algorithm fits suitably to the signalized intersection control problem with bus priority. Three representative traffic parameters were evaluated by the simulations. The parameters of buses improved significantly in all scenarios. The results concerning the private cars are mixed in consequence of the indirect priority measures for buses.

The application of the strategy may be realizable with special infrared detector providing passenger data. Although such systems are not common in traffic engineering practice the problem is solvable technically. Moreover, as a future technology the cellular phone information (based on network cell data) may be used for passenger detection as well. The potentially saved external costs would compensate the investments of such measurement system.

The proposed traffic control needs further researches to investigate the effects of different green time constraints, traffic dependent cycle times or variable stage orders.

\section{Acknowledgements}

The work is connected to the scientific program of the "Development of quality-oriented and harmonized $\mathrm{R}+\mathrm{D}+\mathrm{I}$ strategy and functional model at BME" project. This project is supported by the New Széchenyi Plan (Project ID: TÁMOP4.2.1/B-09/1/KMR-2010-0002). This work is connected to the scientific program of EITKIC-12-1-2012-0001 project (supported by the Hungarian Government, managed by the National Development Agency, financed by the Research and Technology Innovation Fund), and TÁMOP-4.2.2.C-11/1/KONV-20120012: Smarter Transport project (supported by the Hungarian Government, co-financed by the European Social Fund).

\section{References}

1 Arasan V, Vedagiri P, Study of the impact of exclusive bus lane under highly heterogeneous traffic condition, Public Transport, 2, (2010), 135-155, DOI 10.1007/s12469-010-0021-x.

2 Balke KN, Dudek CL, Urbanik T, Development and Evaluation of Intelligent Bus Priority Concept, Transportation Research Record, 1727, (2000), 12-19.

3 Bretherton RD, Hounsell NB, Radia B, Public Transport Priority in SCOOT, In: Proceedings of the 3rd Annual World Congress on ITS Systems, 1996.

4 Cortes CE, Saez D, Milla F, Nunez A, Riquelme M, Hybrid predictive control for real-time optimization of public transport systems operations based on evolutionary multi-objective optimization, Transportation Research Part C, 18, (2010), 257-269.

5 Davol AP, Modeling of traffic signal control and transit signal priority strategies in a microscopic simulation laboratory, Masters thesis, MIT, 2001.

6 Dion F, Rakha $\mathbf{H}$, Zhang $\mathbf{Y H}$, Evaluation of potential transit signal priority benefits along a fixed-time signalized arterial, Journal of Transportation Engineering, 130, (2004), 294-303.

7 Eichler M, Daganzo CF, Bus lanes with intermittent priority: Strategy formulae and an evaluation, Transportation Research Part B: Methodological, 40(9), (2006), 731 - 744

8 Koehler LA, Kraus W, Simultaneous control of traffic lights and bus departure for priority operation, Transportation Research Part C, 18, (2010), 288-298.

9 Liao CF, Davis GA, Simulation Study of a Bus Signal Priority Strategy Based on GPS/AVL and Wireless Communications, In: TRB 2007 Annual Meeting CD-ROM, 2007.

10 Mirchandani P, Head L, Knyazyan A, Wu W, An Approach Towards the Integration of Bus Priority, Traffic Adaptive Signal Control, and Bus Information/Scheduling Systems, In: S. Voss, D. Daduna (eds.), Traffic Adaptive Signal Control, and Bus Information/Scheduling Systems, Springr Verlag, Germany, 2001, pp. 319-334.

11 Mirchandani $\mathbf{P}, \mathbf{L i} \mathbf{J}-\mathbf{Q}$, Hickman M, A macroscopic model for integrating bus signal priority with vehicle rescheduling, Public Transport, 2, (2010), 159-172, DOI 10.1007/s12469-010-0028-3.

12 Pavlidis I, Symosek P, Morellas V, Fritz B, Papanikolopoulos NP, Sfarzo R, Automatic passenger counting in the HOV lane, University of Minnesota, Minnesota Department of Transportation, 1999. MN/RC-200006.

13 Polgár J, Tettamanti T, Varga I, Autóbusz előnybiztosító logika forgalomfüggó irányítású csomópontban (journal paper in Hungarian), Bus priority strategy in traffic dependent intersection control, Városi Közlekedés, 51, (2011), 174-181.

14 Skabardonis A, Control Strategies for Transit Priority, Transportation Research Record, 1727, (2000), 20-26.

15 Vehicle Occupancy Ltd., UK, WWW . vehicleoccupancy.com

16 Wu J, Hounsell N, Bus Priority Using pre-signals, Transportation Research Part A: Policy and Practice, 32(8), (1998), 563 - 583, DOI 10.1016/S09658564(98)00008-1.

17 VISSIM 5.3 COM Interface manual, PTV AG., 2010.

18 VISSIM 5.3 User Manual, PTV AG., 2010. 